\title{
Is It Possible to Develop Cancer Vaccines to Neoantigens, What Are the Major Challenges, and How Can These Be Overcome?
}

\section{Targeting the Right Antigens in the Right Patients}

\author{
Stephen P. Schoenberger \\ Laboratory of Cellular Immunology, La Jolla Institute for Allergy and Immunology, La Jolla, California 92037; \\ Division of Hematology and Oncology, UCSD Moores Cancer Center, San Diego, California 92123; and Cancer \\ Vaccines Group, Human Longevity Inc., San Diego, California 92121 \\ Correspondence: sps@lji.org
}

\begin{abstract}
Recent advances in genomic sequencing and bioinformatics have empowered a revolution in immuno-oncology that has led to numerous unambiguous demonstrations of spontaneous and therapy-induced T-cell responses in patients against a subset of immunogenic tumorspecific somatic mutations known as neoantigens. These findings raise the exciting possibility that patients could be therapeutically treated with personalized vaccines against the mutations expressed by their own tumor. A central challenge for the broader clinical application of this approach will be to define the best antigens to target, to determine the subset of patients most likely to derive significant clinical benefit, and, finally, to discover both the best method of vaccine delivery and the optimal time in the disease course to do so. A growing number of translational immunologists believe that these challenges can be overcome and this perspective will discuss strategies to achieve this.
\end{abstract}

\section{GREAT DEBATES}

What are the most interesting topics likely to come up over dinner or drinks with your colleagues? Or, more importantly, what are the topics that don't come up because they are a little too controversial? In Immune Memory and Vaccines: Great Debates, Editors Rafi Ahmed and Shane Crotty have put together a collection of articles on such questions, written by thought leaders in these fields, with the freedom to talk about the issues as they see fit. This short, innovative format aims to bring a fresh perspective by encouraging authors to be opinionated, focus on what is most interesting and current, and avoid restating introductory material covered in many other reviews.

The Editors posed 13 interesting questions critical for our understanding of vaccines and immune memory to a broad group of experts in the field. In each case, several different perspectives are provided. Note that while each author knew that there were additional scientists addressing the same question, they did not know who these authors were, which ensured the independence of the opinions and perspectives expressed in each article. Our hope is that readers enjoy these articles and that they trigger many more conversations on these important topics.

Editors: Shane Crotty and Rafi Ahmed

Additional Perspectives on Immune Memory and Vaccines: Great Debates available at www.cshperspectives.org

Copyright (C 2018 Cold Spring Harbor Laboratory Press; all rights reserved; doi: 10.1101/cshperspect.a028837

Cite this article as Cold Spring Harb Perspect Biol 2018;10:a028837 
S.P. Schoenberger

t has long been appreciated that cancer is a disease of genes, specifically those that control the life and death of a cell, which sustain function-altering mutations, leading to the formation of tumors that can eventually leave their site of origin and metastasize to new environments within the body (Boveri 2008; Hanahan and Weinberg 2011). The advent of low-cost next-generation sequencing technology coupled with advances in bioinformatics and cellular immunology have in recent years revealed that the same mutational process that is crucial to the development of cancer also creates opportunities for immune recognition of a subset of somatic mutations called neoantigens (Fig. 1) (reviewed in Schumacher and Schreiber 2015). The generation of neoantigen-specific $\mathrm{T}$ cells can occur either spontaneously or as a result of immunotherapeutic intervention, for example, through immune checkpoint blockade (ICB) with antibodies against CTLA-4 or the PD-1/PD-L1 pathway (Rajasagi et al. 2014; Wick et al. 2014; Linnemann et al. 2015;
McGranahan et al. 2016). That such neoantigen-specific T-cell responses can be of significant clinical benefit is inferred by the superior efficacy of ICB in patients bearing tumors with higher levels of somatic mutations, the finding that the therapeutic responses driven by ICB target neoantigens, and the finding that neoantigens are prominent targets of effective adoptive cellular therapy (ACT) (Hodi et al. 2010; Robbins et al. 2013; van Rooij et al. 2013; Lu et al. 2014; Snyder et al. 2014; Tran et al. 2014; Rizvi et al. 2015; McGranahan et al. 2016). Despite this wealth of encouraging data showing that tumor neoantigens offer a unique set of targets for effective personalized immunotherapy, vaccination strategies based on neoantigens are just beginning to be evaluated in human trials (Schlom 2012; Vonderheide and Nathanson 2013). As with any new therapeutic approach, neoantigen vaccination must overcome a number of crucial challenges before its true potential in cancer treatment can be realized.

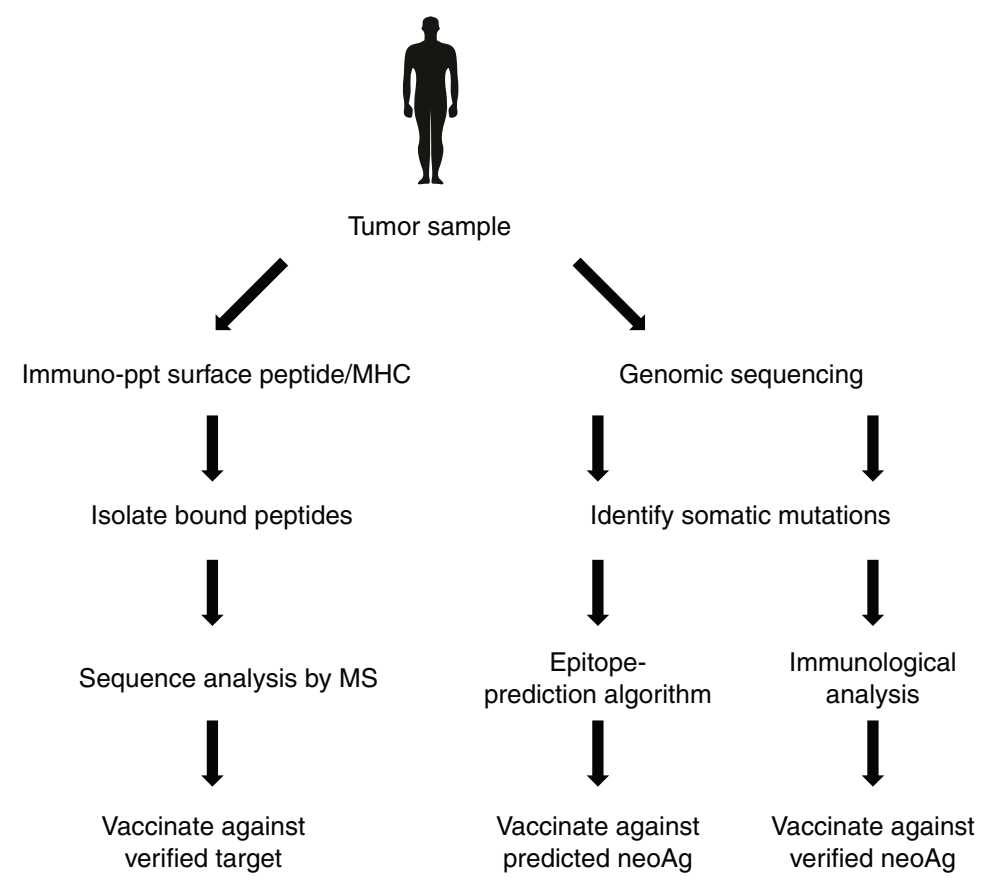

Figure 1. Schematic shows approaches to neoantigen discovery. MHC, Major histocompatibility complex; MS, mass spectrometry; neoAg, neoantigen. 


\section{WHICH ANTIGENS?}

The goal of effective vaccination is the induction of prophylactic or therapeutic immunity against a specific disease or its etiologic agent. The choice of antigens targeted in this is crucial, as they must be selectively presented on the majority of tumor cells in a form suitable for recognition by a corresponding repertoire of host $\mathrm{T}$ cells. Cancer presents a number of challenges in this regard, both at the level of target identification and in their evolution over time. Our ability to identify tumor-expressed somatic mutations has been transformed with the advent of modern massively parallel next-generation sequencing (NGS) to the point where it is now technically and economically feasible to perform routine longitudinal clinical-grade sequencing of tumor exomes and RNA for cancer patients (Khotskaya et al. 2017). Our ability to discern the subset of somatic mutations that can be verified as neoantigens (i.e., those that can be targeted by $\mathrm{T}$ cells for specific recognition of a tumor), however, is significantly less certain. $\mathrm{Al}$ though recent advances in the sensitivity of mass spectrometry has enabled the direct identification of both nonmutated and mutant major histocompatibility complex (MHC)-bound Tcell ligands, this approach may face technical challenges because of the amount of tumor tissue needed and its cellular complexity (Walter et al. 2012; Rammensee and Singh-Jasuja 2013; Yadav et al. 2014). Based on a deeper understanding of the rules governing generation of T-cell ligands, the task of neoantigen prediction has been assisted by the development of an impressive array of computational tools that analyze various features of tumor-specific mutations considered to be relevant for antigen processing and presentation, including their proteasome cleavage, transporter associated with antigen processing (TAP)-mediated endoplasmic reticulum (ER) translocation, and predicted binding affinity for MHC (reviewed in Cherryholmes et al. 2015). While these tools have effectively reduced the number of mutations to be considered for inclusion in a vaccine, their success in predicting which mutations are found to be capable of inducing a T-cell response, which recognizes a tumor cell, in other words, which can be verified as neoantigens, is surprisingly low-on the order of $1 \%-2 \%$ of input expressed mutations-especially when considered from the perspective of the more numerous cancer types, which are characterized by an intermediate/low mutational burden (Alexandrov et al. 2013; Lawrence et al. 2013; Wick et al. 2014). The somewhat pessimistic view emerging from these findings is that, unless a tumor has at least 50-100 expressed somatic mutations, there would appear to be little chance that any of them could be effectively targeted by a neoantigen-specific vaccine (Martin et al. 2016). It is important to consider, however, that this perspective is heavily influenced by the stringent criteria that the in silico algorithms apply to determine which mutations are deemed suitable for functional response testing or inclusion in a neoantigen vaccine, as well as the reliance on single cytokines, usually interferon $\gamma($ IFN- $\gamma$ ) or tumor necrosis factor (TNF) or peptide/MHC multimers as readouts of a functional T-cell repertoire. If, however, tumors can both induce and be recognized by a neoantigen-specific T-cell repertoire that is broader in terms of fine specificity and effector profile than accounted for by current methods, it is conceivable that the actual range of vaccine targets has been underestimated. An alternative to the speculative nature of predictive algorithms would be to directly identify the subset of all expressed mutations that have elicited detectable T-cell responses from a patient's peripheral blood mononuclear cells (PBMCs) through the use of multiparametric ex vivo functional assays, such as those measuring cytokine production, surface marker upregulation, or a combinations of these. It has been suggested that identifying neoantigens through short-term $\mathrm{T}$-cell assays would reveal the repertoire of ineffective T-cell responses in cancer patients (Fritsch et al. 2014). Given the multiple mechanisms through which $\mathrm{T}$ cells are functionally inhibited within the local tumor microenvironment, however, it would seem unlikely that their antigen specificity alone would dictate their capacity for tumor control (reviewed in Motz and Coukos 2013). The neoantigens that have induced a spontaneous response 
within a cancer patient detectable in blood are those that, in a sense, have already "passed the test" of participating in cross-presentation and are being targeted by a functional T-cell repertoire-these would seem to be excellent choices for initial vaccine targets. Among these, there is ample evidence to suggest that both $\mathrm{CD}^{+}$and $\mathrm{CD}^{+}$T-cell epitopes should be included, as both subsets have individual and synergistic effects on tumor control (Melief et al. 2015).

A further level of complexity in leveraging tumor neoantigens through vaccination lies in their clonal heterogeneity of expression. The unprecedented insight into the cancer genome made possible by NGS and bioinformatics has revealed that tumors are in a constant state of dynamic and adaptive clonal evolution as they strive for robust survival in a changing ecosystem (Greaves 2015). This is especially relevant for therapeutic vaccination strategies, as targeting the immune response to subclonal branch mutations rather than the clonal trunk mutations expressed in the majority of tumors would be predicted to lead to treatment failure and the emergence of resistant clones (McGranahan et al. 2016). Whereas it would seem only logical to target the clonal neoantigens for vaccines, their reliable identification may prove difficult as sequencing a single biopsy fails to capture the breadth and hierarchy of somatic mutations expressed in the context of metastatic disease (Gerlinger et al. 2012). One approach to overcome this would be to simply sequence more tumors sites within an individual patient, or sequence different areas within a single biopsy site in an effort to reveal its inherent complexity; both are becoming increasingly feasible as NGS costs decrease (Christensen et al. 2015; Siegmund and Shibata 2016). With respect to which neoantigens to target through vaccination, those occurring in so-called "driver" oncogenes promoting tumor growth and metastasis have long been seen as attractive as they would be expected to be both clonally expressed and conserved through evolutionary pressure to maintain oncogenic fitness. Given the apparent low frequency with which a given patient's immune system can be expected to respond to a single protein antigen that differs from self by a single residue, and the widely varying intratumoral heterogeneity (IHT) observed across different tumor types and disease states, it would seem a safer prospect to pursue targets of opportunity identified through the ex vivo approach described above (Lawrence et al. 2013; Zhang et al. 2014; Lee et al. 2015). In light of subclonality and the numerous adaptive resistance mechanisms used by tumors, it would additionally seem essential to target several neoantigens simultaneously to avoid loss of efficacy for an otherwise potent $\mathrm{T}$-cell response against a single target epitope (McGranahan et al. 2016; Verdegaal et al. 2016). It follows then that the best approach for antigen selection would include both driver and passenger mutations that display a high degree of clonal expression and for which a responding $\mathrm{T}$-cell repertoire can be shown.

\section{WHICH PATIENTS?}

Advances in our ability to accurately identify patient-specific neoantigens will progressively extend the range of tumor types that can be targeted for vaccination to those at the lower end of the mutational burden spectrum. Whereas this is undoubtedly good news, the full potential of this approach to personalized medicine can be reached by understanding not only what antigens to vaccinate against but also which patients are most likely to benefit. Until proven to improve survival or disease outcome, neoantigen vaccines must first be evaluated as experimental therapeutics for patients with advanced disease for whom few other options exist. Presuming a positive result from the numerous ongoing phase I clinical studies addressing this, the eventual position of neoantigen vaccines within current treatment paradigms becomes a relevant question. Cancer patients can broadly be divided into those with progressive disease who have failed conventional therapy, such as Parkinson's disease (PD), and a numerically larger group who have reached the status of minimalresidual or no evidence of disease (MRD/NED) following a successful therapeutic intervention. The former category presents a difficult task for a therapy that depends on the generation of po- 
tent responses from an immune system that has been compromised by prior systemic chemotherapy and the global and local immune dysregulation that frequently occurs in advanced disease (Shehata and Karim 2014; Camisaschi et al. 2016; Shaked 2016). The latter category (MRD/NED) contains patients whose disease characteristics carry a high risk of relapse, and it is these patients that constitute the most logical cohort to benefit from neoantigen vaccination for a number of reasons. In terms of tissue access, such patients are more likely to have had the opportunity for tumor genomic sequencing and analysis during the course of their initial treatment, it would seem logical that mutational profiling and neonatigen prediction could more easily be performed. Additionally, their performance status and immune responsiveness should be improve following completion of systemic chemotherapy, such that could be expected to mount a stronger response to vaccination. Although IHT could still preclude the goal of sterilizing immunity against all micrometastases, targeting a broad range of epitopes should confer significant protection.

\section{HOW TO VACCINATE?}

The final delivery of neoantigens in a formulation suitable to induce potent $\mathrm{T}$-cell responses remains an area of active clinical investigation. The task is daunting in its complexity and urgency, as it requires the generation of a personalized product in a time frame consistent with providing therapeutic benefit for patients with a progressively growing tumor or one at high risk of recurrence. As such, a building consensus holds that synthetic vaccines such as DNA, RNA, or peptides would be most suitable from the perspective of design, manufacturing, and safety than recombinant viral vectors, although the latter could be modified to enable faster manufacturing timelines through the use of neoantigen cassettes. Engineered bacterial strains such as attenuated Listeria monocytogenes represent a middle ground between the potency of a live pathogen and the rapid production needs of a personalized neoantigen vaccine. Each of these approaches are currently be- ing evaluated in industry-sponsored phase I clinical trials, and the next few years should see the emergence of critical data on their ability to meet immunological versus clinical end points in these early studies. Their chances for success are further heightened by the opportunity for combining them with the growing armamentarium of immune complex (IC)blocking antibodies, which should act as a force multiplier for induction of potent tumor-specific immunity.

The theoretical, experimental, and clinical foundations for vaccines in improving human health are well established. The advent of routine low-cost genomic sequencing of tumors and improved methods of identifying clonally expressed somatic mutations herald the era in which the concepts of vaccinating against that which is foreign can be applied to engineering a cure from within.

\section{REFERENCES}

Alexandrov LB, Nik-Zainal S, Wedge DC, Aparicio SAJR, Behjati S, Biankin AV, Bignell GR, Bolli N, Borg A, Børresen-Dale AL, et al. 2013. Signatures of mutational processes in human cancer. Nature 500: 415-421.

Boveri T. 2008. Concerning the origin of malignant tumours by Theodor Boveri. Translated and annotated by Henry Harris. J Cell Sci 121: 1-84.

Camisaschi C, Vallacchi V, Vergani E, Tazzari M, Ferro S, Tuccitto A, Kuchuk O, Shahaj E, Sulsenti R, Castelli C, et al. 2016. Targeting immune regulatory networks to counteract immune suppression in cancer. Vaccines (Basel) 4: 38.

Cherryholmes GA, Stanton SE, Disis ML. 2015. Current methods of epitope identification for cancer vaccine design. Vaccine 33: 7408-7414.

Christensen KD, Dukhovny D, Siebert U, Green RC. 2015. Assessing the costs and cost-effectiveness of genomic sequencing. J Pers Med 5: 470-486.

Fritsch EF, Rajasagi M, Ott PA, Brusic V, Hacohen N, Wu CJ. 2014. HLA-binding properties of tumor neoepitopes in humans. Cancer Immunol Res 2: 522-529.

Gerlinger M, Rowan AJ, Horswell S, Larkin J, Endesfelder D, Gronroos E, Martinez P, Matthews N, Stewart A, Tarpey $\mathrm{P}$, et al. 2012. Intratumor heterogeneity and branched evolution revealed by multiregion sequencing. $N$ Engl J Med 366: 883-892.

Greaves M. 2015. Evolutionary determinants of cancer. Cancer Discov 5: 806-820.

Hanahan D, Weinberg RA. 2011. Hallmarks of cancer: The next generation. Cell 144: 646-674.

Hodi FS, O’Day SJ, McDermott DF, Weber RW, Sosman JA Haanen JB, Gonzalez R, Robert C, Schadendorf D, Hassel 
S.P. Schoenberger

JC, et al. 2010. Improved survival with ipilimumab in patients with metastatic melanoma. N Engl J Med 363: 711-723.

Khotskaya YB, Mills GB, Mills Shaw KR. 2017. Next-generation sequencing and result interpretation in clinical oncology: Challenges of personalized cancer therapy. Annu Rev Med 68: 113-125.

Lawrence MS, Stojanov P, Polak P, Kryukov GV, Cibulskis K, Sivachenko A, Carter SL, Stewart C, Mermel CH, Roberts SA, et al. 2013. Mutational heterogeneity in cancer and the search for new cancer-associated genes. Nature 499: 214-218.

Lee JY, Yoon JK, Kim B, Kim S, Kim MA, Lim H, Bang D, Song YS. 2015. Tumor evolution and intratumor heterogeneity of an epithelial ovarian cancer investigated using next-generation sequencing. BMC Cancer 15: 85 .

Linnemann C, van Buuren MM, Bies L, Verdegaal EME, Schotte R, Calis JJA, Behjati S, Velds A, Hilkmann H, Atmioui DE, et al. 2015. High-throughput epitope discovery reveals frequent recognition of neo-antigens by $\mathrm{CD} 4^{+} \mathrm{T}$ cells in human melanoma. Nat Med 21: 81-85.

Lu Y-C, Yao X, Crystal JS, Li YF, El-Gamil M, Gross C, Davis L, Dudley ME, Yang JC, Samuels Y, et al. 2014. Efficient identification of mutated cancer antigens recognized by $\mathrm{T}$ cells associated with durable tumor regressions. Clin Cancer Res 20: 3401-3410.

Martin SD, Brown SD, Wick DA, Nielsen JS, Kroeger DR, Twumasi-Boateng K, Holt RA, Nelson BH. 2016. Low mutation burden in ovarian cancer may limit the utility of neoantigen-targeted vaccines. PLOS ONE 11: e0155189.

McGranahan N, Furness AJS, Rosenthal R, Ramskov S, Lyngaa R, Saini SK, Jamal-Hanjani M, Wilson GA, Birkbak NJ, Hiley CT, et al. 2016. Clonal neoantigens elicit T cell immunoreactivity and sensitivity to immune checkpoint blockade. Science 351: 1463-1469.

Melief CJM, van Hall T, Arens R, Ossendorp F, van der Burg SH. 2015. Therapeutic cancer vaccines. J Clin Invest 125: 3401-3412.

Motz GT, Coukos G. 2013. Deciphering and reversing tumor immune suppression. Immunity 39: 61-73.

Rajasagi M, Shukla SA, Fritsch EF, Keskin DB, DeLuca D, Carmona E, Zhang W, Sougnez C, Cibulskis K, Sidney J, et al. 2014. Systematic identification of personal tumorspecific neoantigens in chronic lymphocytic leukemia. Blood 124: 453-462.

Rammensee HG, Singh-Jasuja H. 2013. HLA ligandome tumor antigen discovery for personalized vaccine approach Expert Rev Vaccines 12: 1211-1217.

Rizvi NA, Hellmann MD, Snyder A, Kvistborg P, Makarov V, Havel JJ, Lee W, Yuan J, Wong P, Ho TS, et al. 2015 Cancer immunology. Mutational landscape determines sensitivity to PD-1 blockade in non-small cell lung cancer. Science 348: 124-128.

Robbins PF, Lu YC, El-Gamil M, Li YF, Gross C, Gartner J, Lin JC, Teer JK, Cliften P, Tycksen E, et al. 2013. Mining exomic sequencing data to identify mutated antigens recognized by adoptively transferred tumor-reactive $\mathrm{T}$ cells. Nat Med 19: 747-752.
Schlom J. 2012. Therapeutic cancer vaccines: Current status and moving forward. J Natl Cancer Inst 104: 599-613.

Schumacher TN, Schreiber RD. 2015. Neoantigens in cancer immunotherapy. Science 348: 69-74.

Shaked Y. 2016. Balancing efficacy of and host immune responses to cancer therapy: The yin and yang effects. Nat Rev Clin Oncol 13: 611-626.

Shehata MA, Karim NA. 2014. Influenza vaccination in cancer patients undergoing systemic therapy. Clin Med Insights Oncol 8: 57-64.

Siegmund K, Shibata D. 2016. At least two well-spaced samples are needed to genotype a solid tumor. BMC Cancer 16: 250 .

Snyder A, Makarov V, Merghoub T, Yuan J, Zaretsky JM, Desrichard A, Walsh LA, Postow MA, Wong P, Ho TS, et al. 2014. Genetic basis for clinical response to CTLA-4 blockade in melanoma. N Engl J Med 371: 2189-2199.

Tran E, Turcotte S, Gros A, Robbins PF, Lu Y-C, Dudley ME, Wunderlich JR, Somerville RP, Hogan K, Hinrichs CS, et al. 2014. Cancer immunotherapy based on mutation-specific $\mathrm{CD} 4^{+} \mathrm{T}$ cells in a patient with epithelial cancer. Science 344: 641-645.

van Rooij N, van Buuren MM, Philips D, Velds A, Toebes M, Heemskerk B, van Dijk LJA, Behjati S, Hilkmann H, Atmioui El D, et al. 2013. Tumor exome analysis reveals neoantigen-specific T-cell reactivity in an ipilimumabresponsive melanoma. J Clin Oncol 31: e439-e442.

Verdegaal EME, de Miranda NFCC, Visser M, Harryvan T, van Buuren MM, Andersen RS, Hadrup SR, van der Minne CE, Schotte R, Spits H, et al. 2016. Neoantigen landscape dynamics during human melanoma-T cell interactions. Nature 536: 91-95.

Vonderheide RH, Nathanson KL. 2013. Immunotherapy at large: The road to personalized cancer vaccines. Nat Med 19: 1098-1100.

Walter S, Weinschenk T, Stenzl A, Zdrojowy R, Pluzanska A, Szczylik C, Staehler M, Brugger W, Dietrich P-Y, Mendrzyk R, et al. 2012. Multipeptide immune response to cancer vaccine IMA901 after single-dose cyclophosphamide associates with longer patient survival. Nat Med 18: 12541261.

Wick DA, Webb JR, Nielsen JS, Martin SD, Kroeger DR, Milne K, Castellarin M, Twumasi-Boateng K, Watson PH, Holt RA, et al. 2014. Surveillance of the tumor mutanome by $\mathrm{T}$ cells during progression from primary to recurrent ovarian cancer. Clin Cancer Res 20: 1125-1134.

Yadav M, Jhunjhunwala S, Phung QT, Lupardus P, Tanguay J, Bumbaca S, Franci C, Cheung TK, Fritsche J, Weinschenk T, et al. 2014. Predicting immunogenic tumour mutations by combining mass spectrometry and exome sequencing. Nature 515: 572-576.

Zhang J, Fujimoto J, Zhang J, Wedge DC, Song X, Zhang J, Seth S, Chow CW, Cao Y, Gumbs C, et al. 2014. Intratumor heterogeneity in localized lung adenocarcinomas delineated by multiregion sequencing. Science 346: 256259. 


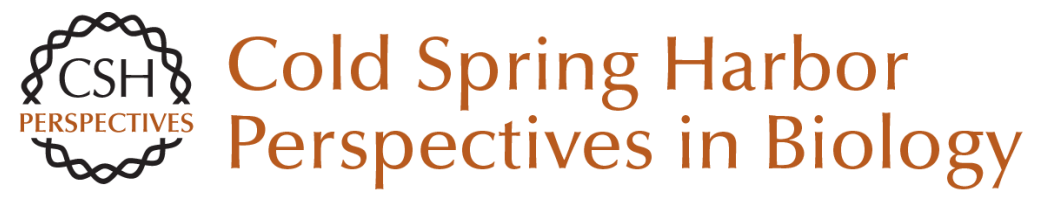

\title{
Is It Possible to Develop Cancer Vaccines to Neoantigens, What Are the Major Challenges, and How Can These Be Overcome?: Targeting the Right Antigens in the Right Patients
}

\author{
Stephen P. Schoenberger \\ Cold Spring Harb Perspect Biol 2018; doi: 10.1101/cshperspect.a028837 originally published online \\ December 18, 2017
}

Subject Collection Immune Memory and Vaccines: Great Debates

Is There Natural Killer Cell Memory and Can It Be Harnessed by Vaccination?: Can Natural Killer and CD8 T Cells Switch Jobs?

Christine A. Biron and Marcus Altfeld

Is There Natural Killer Cell Memory and Can It Be Harnessed by Vaccination?: Vaccination Strategies Based on NK Cell and ILC Memory Megan A. Cooper, Todd A. Fehniger and Marco Colonna

Is It Possible to Develop Cancer Vaccines to Neoantigens, What Are the Major Challenges, and How Can These Be Overcome?: Neoantigens as Vaccine Targets for Cancer Haydn T. Kissick

Is It Possible to Develop Cancer Vaccines to Neoantigens, What Are the Major Challenges, and How Can These Be Overcome?: Neoantigens: Nothing New in Spite of the Name

Olivera J. Finn and Hans-Georg Rammensee
Is There Natural Killer Cell Memory and Can It Be Harnessed by Vaccination?: NK Cell Memory and Immunization Strategies against Infectious Diseases and Cancer Joseph C. Sun and Lewis L. Lanier

Is There Natural Killer Cell Memory and Can It Be Harnessed by Vaccination?: Natural Killer Cells in Vaccination

Harold R. Neely, Irina B. Mazo, Carmen Gerlach, et al.

Is It Possible to Develop Cancer Vaccines to Neoantigens, What Are the Major Challenges, and How Can These Be Overcome?: Targeting the Right Antigens in the Right Patients Stephen P. Schoenberger

Which Dengue Vaccine Approach Is the Most Promising, and Should We Be Concerned about Enhanced Disease after Vaccination?: There Is Only One True Winner Scott B. Halstead

For additional articles in this collection, see http://cshperspectives.cshlp.org/cgi/collection/

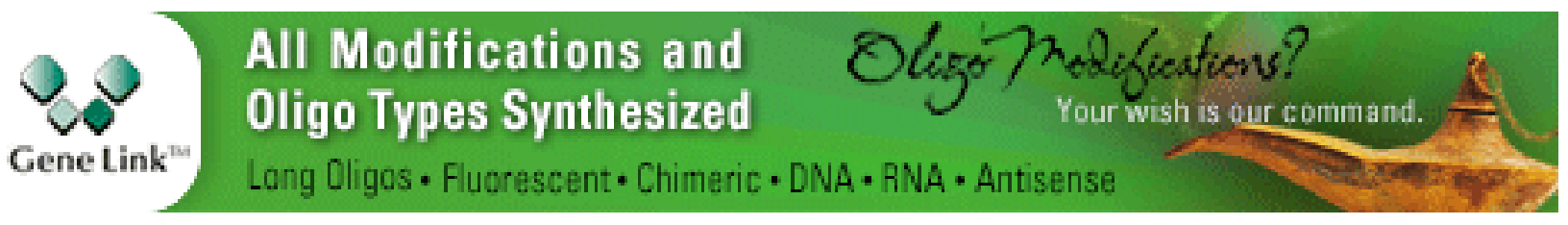


Which Dengue Vaccine Approach Is the Most Promising, and Should We Be Concerned about Enhanced Disease after Vaccination?: The Challenges of a Dengue Vaccine

Gavin Screaton and Juthathip Mongkolsapaya

Which Dengue Vaccine Approach Is the Most Promising, and Should We Be Concerned about Enhanced Disease after Vaccination?: The Path to a Dengue Vaccine: Learning from Human Natural Dengue Infection Studies and Vaccine Trials Aravinda M. de Silva and Eva Harris

Is It Possible to Develop a "Universal" Influenza Virus Vaccine?: Potential for a Universal Influenza Vaccine James E. Crowe, Jr.

Is It Possible to Develop a "Universal" Influenza Virus Vaccine?: Outflanking Antibody Immunodominance on the Road to Universal Influenza Vaccination

Davide Angeletti and Jonathan W. Yewdell
Which Dengue Vaccine Approach Is the Most Promising, and Should We Be Concerned about Enhanced Disease after Vaccination?: Questions Raised by the Development and Implementation of Dengue Vaccines: Example of the Sanofi Pasteur Tetravalent Dengue Vaccine Bruno Guy

Which Dengue Vaccine Approach Is the Most Promising, and Should We Be Concerned about Enhanced Disease after Vaccination?: The Risks of Incomplete Immunity to Dengue Virus Revealed by Vaccination

Stephen S. Whitehead and Kanta Subbarao

Is It Possible to Develop a "Universal" Influenza Virus Vaccine?: Immunogenetic Considerations Underlying B-Cell Biology in the Development of a Pan-Subtype Influenza A Vaccine Targeting the Hemagglutinin Stem Sarah F. Andrews, Barney S. Graham, John R. Mascola, et al.

Is It Possible to Develop a "Universal" Influenza Virus Vaccine?: Potential Target Antigens and Critical Aspects for a Universal Influenza Vaccine Florian Krammer, Adolfo García-Sastre and Peter Palese

For additional articles in this collection, see http://cshperspectives.cshlp.org/cgi/collection/

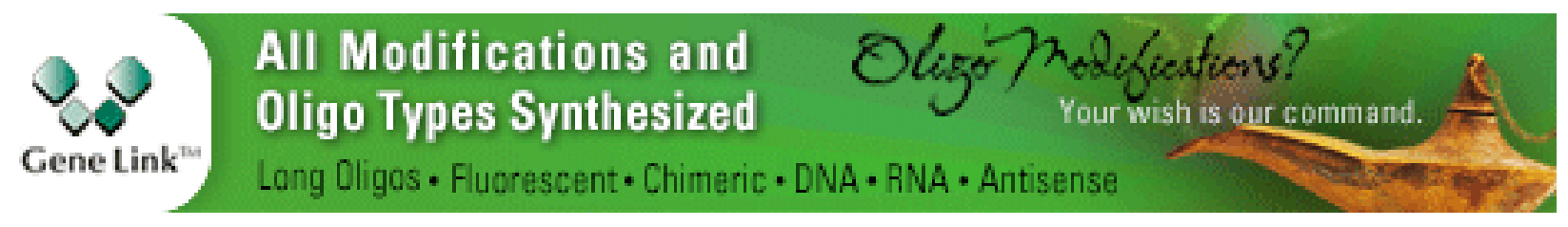

